\title{
A PLS blockchain for loT applications: protocols and architecture
}

\author{
Alex Shafarenko (1D
}

\begin{abstract}
This paper proposes an architecture and a protocol suite for a permissioned blockchain for a local loT network. The architecture is based on a sealed Sequencer and a Fog Server running (post-quantum) Guy Fawkes protocols. The blocks of the blockchain are stored in networked Content Addressable Storage alongside any user data and validity proofs. We maintain that a typical loT device can, despite its resource limitations, use our blockchain protocols directly, without a trusted intermediary. This includes posting and monitoring transactions as well as off-chain (post-quantum) emergency communications without an explicit public key.
\end{abstract}

Keywords: Blockchain, Guy Fawkes protocol, Post-quantum, HORS-OTS, LoRa, Concurrent transmission

\section{Introduction}

This article presents a Block Chain construction based on the well-known Guy Fawkes Protocol (GFP) (Anderson et al. 1998) for digital signature, which we extend and bring to bear on Block Chain (BC) technology intended for a swarm of low-power devices (IoT things). The primary purpose of this blockchain is to support an immutable distributed ledger that ensures the authenticity and sequencing of user records posted on it. Financial transactions for IoT are not our intention, but they should be compatible with our approach.

Motivation We set ourselves the following design constraints on behalf of the participating things:

1. Post Quantum restriction, in particular no public key crypto.

2. Low power, low energy. Notice that the avoidance of public key crypto is synergetic with this constraint.

3. Low local storage. A thing may have a flash card embedded in it, but the use of the flash card eats into the energy budget.

4. Local communications with low bandwidth and short messages. The security protocol should be

Correspondence: A.Shafarenko@herts.ac.uk

Department of Computer Science, University of Hertfordshire, Hatfield AL10 9AB, UK conducted via UHF broadcasts over the target area. An effective adversary should have to radiate significant power (reliably over the legal limit) and expose itself to triangulation.

5. It must be possible for all things to authenticate the ledger under realistic assumptions without relying on a trusted intermediary.

The above constraints have not, to the best of our knowledge, been considered together, but are characteristic of low-end IoT devices. The Post Quantum restriction is currently a bonus (due to insufficient power of available quantum computing), but will doubtless become important at some point in not so distant future.

Challenge We believe the main security risk with our scheme is the Denial of Service (DoS) attack. Due to the use of local communications (constraint 4 above), DoS attacks only need to be impeded but not totally suppressed. The latter is impossible due to the possibility of an attacker's physically jamming the communication infrastructure.

DoS is our only concern for the following reasons. Our proposed protocol does not permit an imposter to impersonate an IoT device to the blockchain manager provided that the device was correctly enrolled on the blockchain and is somehow made physically secure. 
Another possible threat is a weaker version of manin-the-middle whereby the man in the middle does not attempt to convince the legitimate recipient that he is the legitimate sender; he instead receives the sender's broadcast (i.e. radio) message while simultaneously jamming the sender's signal so as to convince the legitimate recipient that no message has been sent yet. The man in the middle then uses the received message to defeat the protocol and impersonate (spoof) the legitimate sender to the legitimate recipient. We call such attacks jam/spoof attacks (more detail given in the section "Original GFP") and propose a countermeasure (see the section "Posting on the blockchain").

\section{Contributions of the paper:}

- We have proposed a Guy-Fawkes type protocol as a basis for a blockchain construction. We show that the protocol achieves authentication, non-repudiation and secure sequencing of the blockchain blocks by itself (not relying on block content). The protocol does not require public key crypto or high-volume communication, both important for an IoT device that is limited in its compute power and communication duty cycle.

- We use the principles of GFP to construct a protocol for an IoT device to post on the blockchain, again without public key crypto, but with guaranteed authenticity and non-repudiation and similarly low communication costs.

- Since the protocol by itself does not protect against DoS attacks we further proposed a network architecture which reduces DoS exposure by exploiting the physical properties of a mainstream communication format: LoRa.

- Finally, since our focus is on the IoT applications, we have constructed an off-chain emergency communication mode that provides authentication and non-repudiation without communicating or storing additional authenticated key material and again, without public key crypto. This is achieved by re-using nonces from the main protocol. As a result each thing is able to send an emergency message between two blocks of the blockchain while enabling any observer of the blockchain to prove the authenticity and provenance of the emergency message based solely on the already published blocks.

Structure The section "Basic protocols" introduces the blockchain protocol. The section "System architecture" defines the system architecture. The protocol for posting content on the blockchain is presented and discussed in the section "Posting on the blockchain". In the subsequent section, the section "Enrolment and optimisations", we show how a user can be enrolled on the chain at a point other than the beginning. The next section puts forward a solution for emergency communications, when the originator cannot wait for the next block of the blockchain to be published. The section "Related work" cites some related work and finally there are conclusions.

\section{Basic protocols}

The Guy Fawkes Protocol family was first proposed by a Cambridge group (Anderson et al. 1998) 10 years ahead of Nakamoto. The original formulation is very clear and can be practically useful, but it was not specifically intended as a blockchain protocol. We will summarise it here for ease of reference, but we still recommend (Anderson et al. 1998) as it contains some important background and a useful discussion.

\section{Original GFP}

The original GFP is a protocol intended for signing messages $M_{i}, i=1,2, \ldots$ using a series of secret codewords $X_{i}=0,1, \ldots$, which are revealed one after another as the protocol progresses. The initial codeword, $X_{0}$, is authenticated out of band when it is revealed, e.g. by digital signature.

The GFP is defined inductively as an unlimited series of rounds. At any round $i \geq 0$ :

1. Select a random codeword $X_{i+1}$ and keep it confidential.

2. Form its hash $h\left(X_{i+1}\right)$

3. Publish $Z_{i+1}=h\left(M_{i+1}, h\left(X_{i+1}\right), X_{i}\right)$

4. Reveal the hash pre-image: $M_{i+1}, h\left(X_{i+1}\right)$, and $X_{i}$

Here $h(\cdot)$ is a cryptographic hash. The security properties of the GFP are based on the computational hardness of fitting a pre-image to a known hash, which is also the basis of, for example, the well-known proof-of-work for the Bitcoin blockchain, and is believed to be robust for a strong enough hash function $h(\cdot)$ even Post Quantum.

How does it work? As this is a signature protocol, it is supposed to ensure that only the originator can run it successfully to sign their messages. The originator is differentiated by their knowledge of $X_{0}$ and, by induction, of every other $X_{i}$, since the introduction of $X_{i+1}$ in step 1 of any round is accompanied by the disclosure of $X_{i}$ in step 4 of the same round. The verifier at step 4 is able to verify that the originator is who they claim they are because it is cryptographically hard to fit the pre-image $\left(M_{i+1}, h\left(X_{i+1}\right), X_{i}\right)$ to its previously published hash $Z_{i+1}$, which means that it is the same pre-image that was used by the actor that published $Z_{i+1}$. Since a part of that preimage is $X_{i}$, which is the pre-image of the hash $h\left(X_{i}\right)$ published as step 4 of the previous round, $i-1$, we conclude by the same logic, that the actor is the same as one present at round $i-1$. We now claim by induction that the 
actor revealing the pre-image in step 4 round $i$ is the same as that in round 0 . However, to participate in round 0 , the actor has to know the signed value of $X_{0}$ before that round, and only the legitimate originator does. This proves the provenance of all messages $M_{i}$ and excludes repudiation.

However, the protocol is phrased in terms of "publish" and "reveal", and as it often happens, the definition of these terms can leave loopholes that nullify the security properties.

Jam/spoof attack. The definitive paper (Anderson et al. 1998) treats step 3 non-specifically and gives newspaper advert publishing as an example of what is meant by "publish". The tacit assumption here is that whatever the originator publishes will be seen by verifiers with certainty before the step- 4 message is revealed. Therein lies the protocol's main vulnerability.

Imagine a situation when an adversary is able to prevent the verifier from receiving the newspaper in question, but the adversary receives it. It then sends the counterfeit copy of the newspaper, in which the message is not included, to a verifier. The verifiers will be unaware that the step3 message has been published. When the step- 4 message is revealed by the originator, the adversary learns $X_{i}$ and is able to publish their own step- 3 message impersonating the originator and using the value of $X_{i}$ learned from the originator when the latter revealed it. Then the adversary is able to send a step- 4 message consistent with that step-3 message and from this time on the current and all subsequent messages are compromised. ${ }^{1}$

We call this the jam/spoof attack. Its existence highlights the fact that the verb "publish" in the protocol description means not only to send a message to the world, but also to guarantee that it has been received. Which is how "publish" differs there from the verb "reveal", which assumes no such guarantee. The verifier expects to see a reveal message after it has received a publish message. If the reveal message is not received before the next publish message, the verifier will simply request one and will keep requesting it until it receives the correct content (the pre-image of the publish message).

The metaphor of newspaper is ideal for this kind of "publish" (provided that the verifier can at least access a library copy of each newspaper issue in time for the reveal message). In reality "publishing" is done by network broadcasting, and in the IoT case that is quite often radio broadcasting, where both jamming and spoofing are quite feasible. Finally, it is interesting to observe that the jam/spoof attack is of the same kind as the classic manin-the-middle attack, since the adversary impersonates the originator to the verifier by placing itself in between,

\footnotetext{
${ }^{1}$ Notice that neither step 3 , nor step 4 require an authenticated channel; in other words, anybody can send these messages and the protocol is supposed to be able to determine which of them come from the genuine counterparty.
}

however the behaviour is different from the classical unauthenticated public-key exchange.

DoS attack. An adversary can exhaust the verifiers' resources without jamming the originator. If $M$ step-3 messages and $N$ step- 4 messages are sent impersonating the originator, the verifier will have to perform up to $M \times N$ verification actions, whereby

i the value $X_{i}$ of the step- 4 message is hashed to match with the value of $h\left(X_{i+1}\right)$ from the previous round. If they don't match, this step- 4 message is invalid, otherwise

ii the whole step- 4 message is hashed to match with at least one step-3 message.

This takes into account the fact that the true step- 4 message can be delayed as it is forwarded through the network, and that the adversary can receive it early. Then a large number of fake step- 4 messages with the correct $X_{i}$ can be produced that pass check (i), making the amount of work closer to the limit $M \times N$.

We will address both attacks in our version of GFP which we present next.

\section{PLS protocol}

We will now introduce a similar construction optimised for our purposes.

Goal Assume that a single transmitter is to broadcast a stream of public, authenticated messages to an unspecified number of receivers. The following conditions must be satisfied:

1. It should be possible (ideally at low cost) for each receiver to prove, without trusting any intermediary, that the message was sent by the transmitter.

2. It should be cryptographically hard for an attacker to modify any message or to change the order of messages without the receivers noticing

3. The broadcaster should be able to send an unlimited number of messages without weakening the security of the previous two constraints (this is common with the original GFP).

Now to the protocol. It uses a standard cryptographic hash $H(x)$ (e.g. SHA-256). All values except $M_{k}$ are binary strings of the same length as $H$. Additionally the protocol uses symmetric encryption $\mathbf{E}_{q}(p)$ which encrypts plaintext $p$ under the key $q$ producing a ciphertext, and its dual decryption: $\mathbf{D}_{q}\left(\mathbf{E}_{q}(p)\right)=p$. This could be any standard cipher, e.g. AES128, suitably adapted to the key and text size using one of the standard methods.

The protocol operates in steps according to the wallclock time. All receivers and the transmitter synchronise 
their clocks so that when the transmitter's clock registers a time $t_{T}$, any receiver's clock $t_{R}$ is no more than $\epsilon$ away:

$$
\left|t_{T}-t_{R}\right|<\epsilon .
$$

The transmitter broadcasts at regular intervals, $t_{0}, t_{0}+$ $\tau, t_{0}+2 \tau, \ldots$, where $\tau \gg \epsilon$. Each broadcast consists of three messages of the same length as $H: P, L$ and $S$; they are a proof, link and signature message, respectively. It is convenient to think of them as being broadcast on three different channels, or in three different time slots, or with a tag that tells the receiver which message it is. The messages are not explicitly indexed, but it is convenient to think of them as being indexed with the Broadcast Interval Number (BIN): BIN 0 corresponds to the interval [ $t_{0}+$ $\left.\epsilon, t_{0}+\tau-\epsilon\right]$, BIN 1 to the interval $\left[t_{0}+\tau+\epsilon, t_{0}+2 \tau-\epsilon\right]$, etc. Note that the $P, L$ and $S$ broadcasts in the same interval are not mutually ordered.

The protocol is presented in Table 1. In or before the first interval, receivers obtain independent authentication of $P_{1}$. In each interval $k$ the transmitter creates a fresh random nonce, $N_{k+1}$, and keeps it secret until the end of the next interval $k+1$. One such nonce, $N_{1}$, is created by the transmitter before launching the protocol.

At every step the transmitter transmit the three messages mentioned above and each receiver attempts to receive them. The link message is saved for the next step and the signature and proof messages are used in the current one. Next the receiver executes the validation procedure detailed in the middle column, which consists of one bitwise XOR calculation and one hash calculation. The calculations involve the current proof message $P_{k}$ and the link message $L_{k-1}$ saved at the previous step. The receiver may have more than one candidate value for $P$ and $L$ which will be obtained by peer communication between receivers. The receiver will subject each $(P, L)$ pair to the validation procedure until it obtains the pair that satisfies it. If no pair passes validation, the protocol fails due to denial of service.

Otherwise the valid $(P, L)$ pair is combined with the current signature message $S_{k}$ to obtain a signed message hash $\sigma_{k}=H\left(M_{k}\right)$. From that time on, the receiver will treat a message whose hash equals $\sigma_{k}$ as having being signed by the transmitter.

\section{Security analysis of PLS \\ Threat model.}

i An attacker can force one or more receivers to receive the attacker's arbitrary message instead of the one being transmitted by the transmitter, or prevent a receiver from receiving the message at all.

ii However, the attacker cannot thus disrupt all receivers and it cannot make any receiver conclude that the broadcast did not take place. The former can be achieved by delivering at least one copy of the broadcast message by an alternative physical channel, and the latter by broadcasting messages on a public wall-clock schedule.

Point (ii) makes it possible for a receiver that the protocol determines has received an invalid message to solicit unauthenticated candidate messages from peer receivers. We will show that using the protocol each receiver will be able to select the genuine message out of a set of candidates. This makes threat (i) a DoS threat rather than a data-integrity one.

We continue with Table 1.

Table 1 PLS Protocol

\begin{tabular}{|c|c|c|c|}
\hline BIN & Transmit/Receive & Verify & Obtain \\
\hline \multirow[t]{3}{*}{1} & $L_{1}=H\left(N_{2}\right) \oplus N_{1}$ & & \\
\hline & $S_{1}=\mathbf{E}_{N_{1}}\left(H\left(M_{1}\right) \oplus H\left(N_{2}\right)\right)$ & $P_{1}$ out of band & \\
\hline & $P_{1}=H\left(N_{1}\right)$ & & \\
\hline \multirow[t]{3}{*}{2} & $L_{2}=H\left(N_{3}\right) \oplus N_{2}$ & & \\
\hline & $S_{2}=\mathbf{E}_{N_{2}}\left(H\left(M_{2}\right) \oplus H\left(N_{3}\right)\right)$ & $H\left(L_{1} \oplus P_{2}\right)=P_{1}$ & $H\left(M_{1}\right)=P_{2} \oplus \mathbf{D}_{L_{1} \oplus P_{2}} S_{1}$ \\
\hline & $P_{2}=H\left(N_{2}\right)$ & & \\
\hline \multirow[t]{3}{*}{3} & $L_{3}=H\left(N_{4}\right) \oplus N_{3}$ & & \\
\hline & $S_{3}=\mathbf{E}_{N_{3}}\left(H\left(M_{3}\right) \oplus H\left(N_{4}\right)\right)$ & $H\left(L_{2} \oplus P_{3}\right)=P_{2}$ & $H\left(M_{2}\right)=P_{3} \oplus \mathbf{D}_{L_{2} \oplus P_{3}} S_{2}$ \\
\hline & $P_{3}=H\left(N_{3}\right)$ & & \\
\hline$\cdots$ & $\cdots$ & $\cdots$ & $\cdots$ \\
\hline \multirow[t]{3}{*}{ k } & $L_{k}=H\left(N_{k+1}\right) \oplus N_{k}$ & & \\
\hline & $S_{k}=\mathbf{E}_{N_{k}}\left(H\left(M_{k}\right) \oplus H\left(N_{k+1}\right)\right)$ & $H\left(L_{k-1} \oplus P_{k}\right)=P_{k-1}$ & $H\left(M_{k-1}\right)=P_{k} \oplus \mathbf{D}_{L_{k-1}} \oplus P_{k} S_{k-1}$ \\
\hline & $P_{k}=H\left(N_{k}\right)$ & & \\
\hline
\end{tabular}


At $\mathrm{BIN}=1$, the transmitter sends out the link, signature and proof messages, saves $N_{2}$ and keeps it secret till the end of the next interval. The receiver receives and saves the received messages. It uses the remaining time in the interval to poll its peers to learn any alternative values of $L, S$, and $P$ should they be received (which may be due to signal propagation problems, deliberate jamming or a cyber attack).

At $\mathrm{BIN}=2$, the receiver receives $P_{2}$ (possibly more than one candidate value)and verifies that for some candidates $L_{1}$ and $P_{2}, H\left(L_{1} \oplus P_{2}\right)=P_{1}$. It means that these values of $L_{1}$ and $P_{2}$ are genuine. Indeed, an attacker wishing to convince the receiver that an alternative link message

$$
\hat{L}_{1}=H\left(\hat{N}_{2}\right) \oplus N_{1} \neq L_{1}
$$

is genuine in order to make a forged signature for its own message $\hat{M}_{1}$

$$
\hat{S}_{1}=\mathbf{E}_{N_{1}}\left(H\left(\hat{M}_{1}\right) \oplus H\left(\hat{N}_{2}\right)\right)
$$

would have to have sent these messages at $\mathrm{BIN}=1$, when only the transmitter knows the value of $N_{2}$, so the attacker would have to use its own nonce, $\hat{N}_{2} \neq N_{2}$, and then force the receiver to receive $\hat{P}_{2}=H\left(\hat{N}_{2}\right)$. To succeed at that, the attacker must be able to obtain $N_{1}$ within interval 1 , to use it in the $\hat{L}_{1}$ message. But all that is publicly known about $N_{1}$ then is its hash, $H\left(N_{1}\right)=P_{1}$.

That is the linchpin of the security of any Guy Fawkes protocol, our version or the classic (Anderson et al. 1998) alike. The attacker has to find a pre-image of a public hash value in order to mount a successful attack. The chances of finding a pre-image are slim, $2^{-(l-1)}$; for $l=256$ the attacker would have to do around $10^{77}$ hash calculations to find the pre-image on a classical computer. This can be improved upon by quantum computing, reducing the number to $2^{-l / 2} \approx 10^{38}$, still reliably unfeasible and certainly good enough for the application domain of the IoT.

Leaving the DoS scenario aside, we assume that the receivers succeed. Next the receiver uses the formula in the last column of Table 1 to calculate $H\left(M_{1}\right)$ on all candidate $S$-messages recorded at $\mathrm{BIN}=1$. All these values are considered equally valid, but only one of them, namely the genuine value of $H\left(M_{1}\right)$, where $M_{1}$ is a message known to the transmitter at $\mathrm{BIN}=1$, can ever be used. An attacker corrupting message $S_{1}$ can only achieve denial of service: to fit a message to an arbitrary hash value of it (for example, by extending the message with a non-data-bearing tail) is computationally as difficult as it is to fit $N_{1}$ to a known $H\left(N_{1}\right)$.

The reader may wonder about the purpose of encryption. In the original Guy Fawkes protocol only hashes are used, but at least four hash-length items need to be communicated in each signing round, whereas PLS requires only three, a $25 \%$ saving in communication costs.
Communication is important for the IoT world, where typically the radio duty cycle of a thing is limited to a fraction of $1 \%$. Also we argue that the computational cost of a hardware-accelerated symmetric encryption (which tends to be AES) is several times cheaper than that of a hash for at least some popular IoT platforms, see the section "Related work" for a specific example and possible reasons.

Another question is whether the public availability of $H\left(M_{1}\right)$ gives the attacker an alternative method for obtaining $N_{1}$ at $\mathrm{BIN}=1$ : by brute-forcing the encryption key. The answer is that this would require the plaintext (the cipher text is publicly available as $S_{1}$ at this point), and to obtain the plaintext the attacker needs to know $H\left(N_{2}\right)=P_{2}$, which is not revealed at $\mathrm{BIN}=1$. To obtain $H\left(N_{2}\right)$ from the public value of $L_{1}$, the attacker requires the value of $N_{1}$ which is what the attacker is trying to fit.

At the end of interval 2 the receiver will have received, and collected from peers all alternative versions of, $L_{2}$ and $S_{2}$ and is now prepared for the next, third interval, etc. The protocol is run periodically as long as the transmitter stays in commission. Since any secrets have a short lifetime $2 \tau$ after which they are published rather than merely not used, there is no accumulation of confidential material at the transmitter site; consequently the transmitter has no security motivated expiration time.

\section{System architecture}

The PLS protocol described above is well suited to serve as a basis for a blockchain system. The key property that makes it so suitable is provable, time-referenced forward chaining, the fact that $L$-messages establish a cryptographically protected, unsplittable temporal chain of $\left\{M_{i}\right\}$, with each $M_{i}$ being defined by its hash $H\left(M_{i}\right)$. With the head of the chain independently authenticated for all actors before the protocol launch, the set of link/proof pairs and the hardness of the hash pre-image problem guarantee that the chain can be validated in isolation by any observer who is present and able to receive messages at broadcast times. No additional source of trust is required to validate the chain although a trusted third party may well be useful as defence against a DoS attack, bearing in mind that light touch security would be sufficient for that purpose.

We propose an architecture of an IoT system with blockchain services, see Fig. 1.

Sequencer At the core of it is placed a physically secure, firmware based, multi-radio connected blockchain Sequencer. The job of the Sequencer is, as the name suggests, building the sequence of chained blocks. The blocks themselves are prepared for the Sequencer by the Fog Server (see below). The Fog Server communicates with the Sequencer in one way (transmits), over a separate authenticated radio channel (Bluetooth, for example), the 


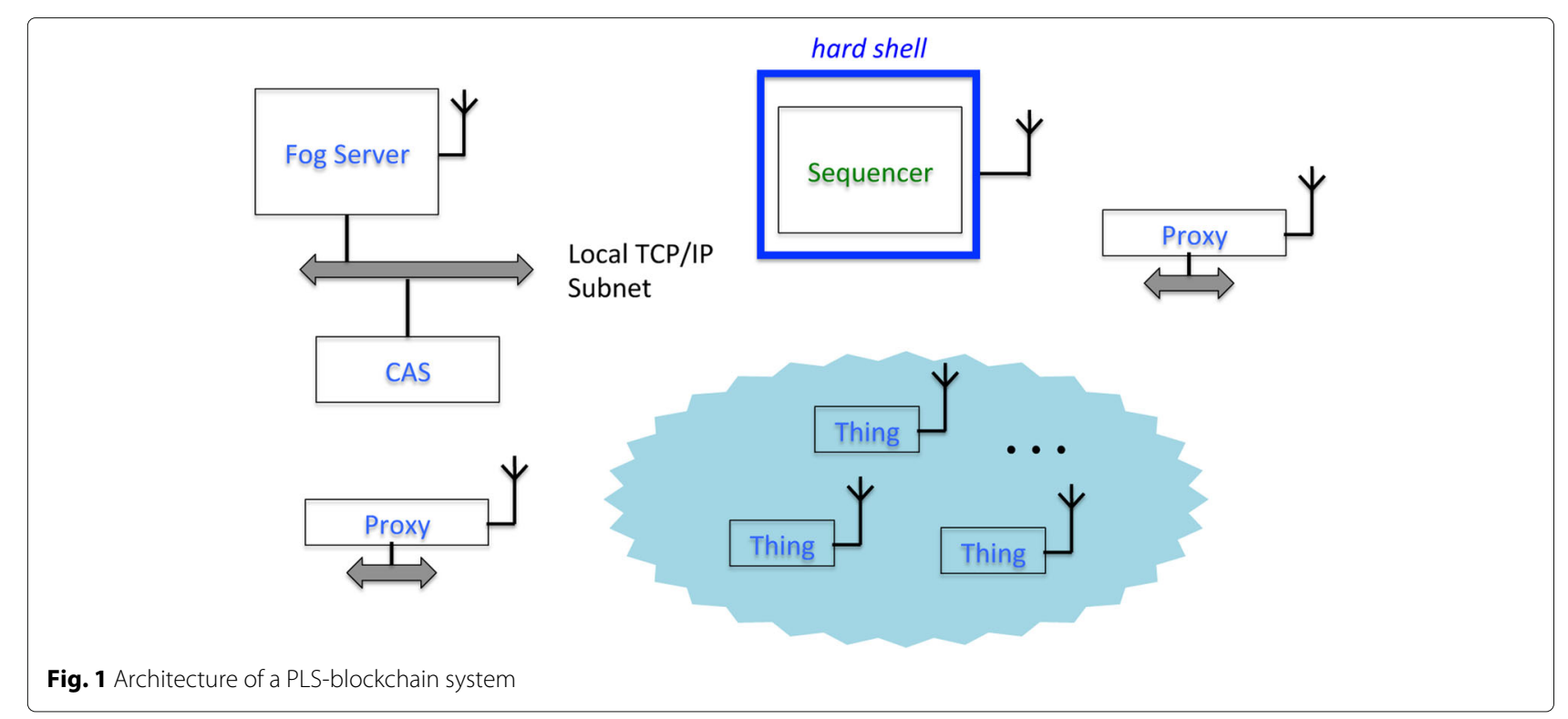

details of which are private to them. What differentiates the Sequencer from the Fog Server is the fact that the Sequencer is not connected to any general purpose networks. Its security role is to run an active PLS protocol on schedule and to keep each nonce $N_{k}$ confidential until the next broadcast period. The Sequencer's embodiment as a separate air-gapped unit with enhanced physical security serves only one purpose: prevention of a blockchain split, which is effective if and only if the confidentiality of $N_{k}$ over a short period of time can be assured. Split avoidance is important for defeating DoS attacks, but the validity of the blocks is not in jeopardy due to the second aspect of the Sequencer, namely the fact that it performs a radio broadcast using a wall-clock synchronised, long-distance signal. As a result, if the defences fail and an attacker compromises the Sequencer, the only non-DoS way to profit from this is to have different broadcasts directed to different groups of things. In this case the inconsistencies will eventually be detected by radio monitoring, but it could be too late, especially if things perform critical work, hence the importance of air-gapping and physical protection for the Sequencer.

CAS. For the purposes of sequencing and signing we are going to use the PLS protocol (and a similar SLVP protocol for things, see the section "Posting on the blockchain"), but what is going to be sequenced and signed are in fact the hashes of the actual blocks and blockchain users' content messages of variable size. To store these items and retrieve them at a user's request, we place a Content-Addressable Storage (CAS) unit on a local TCP/IP subnet and organise a private channel (behind the organisation's firewall) between the unit and the Fog Server. CAS operates in
WORM mode: a file is stored once under the hash of its content as its file name, which means that it cannot be changed without the file name changing, that it is easy to verify that it hasn't been changed and that no trust between a user and the CAS is required. Due to the irreversible nature of hashing, it is prohibitively expensive to store a different content under the same hash, so all the content recipient needs to do to ascertain the integrity of the content is check that its hash is correct.

Every time a new block is formed to go on the block chain, the block content is stored in CAS and the hash of it is used in the protocol. All things have access to CAS via the Server or its Proxy and can retrieve files whenever the content hash is known.

Note that the hash functions used by CAS and our protocols do not have to be the same. By extending the encryption in protocol $S$-messages to accommodate a longer plaintext (which would require expanding $H\left(N_{k+1}\right)$ by replication and concatenation), one could use longer hashes for storage than those used in the protocol. It may be profitable to do so given that the second pre-image resistance of a hash in the PLS protocol need only to withstand an attack over $2 \tau$, while the resistance of the hash function used to compute CAS file names is potentially required to be much greater (years if not decades before broken). This is not an argument for a longer hash for CAS (a standard SHA-256 is quite adequate even against quantum attacks) but perhaps a shorter hash for PLS (128 bits) could be sufficient. This can be achieved easily, by just taking the first 128 bits from the 256 bit output of the SHA-256 hash and ignoring the rest. The shortening is possible due to the fact that security properties of a correctly designed hash function are assured on a per-bit 
basis for the output value; so a subset of the output bits has the same properties as the full set except the collision frequency ${ }^{2}$ (vulnerability to a pre-image attack).

Fog Server. An IoT system's security cannot be completely decentralised since, ultimately, the network of things is owned by an organisation, and since that organisation should have authority to add and remove things, configure them, assign jobs to them, etc. at any time and without waiting for a slow validation process characteristic of most blockchains. This does not necessarily mean that everything should be centralised; in fact very little centralisation is required: mainly the issues of reconfiguration (adding/removing) and conflict resolution (proof service) and possibly running smart contracts on the blockchain, which we will not discuss here.

The presence of the infrastructure above the IoT can be captured by introducing into our architecture an actor that we call the Fog Server (FS), which is a local datacenter that has direct connection to things via a suitable radio network (e.g. LoRa), as well as a sufficient compute power, storage and connection to Cloud. The blockchain users should trust the FS for:

- enrolment of new things to the block chain, and their removal. At the point of enrolment, the Cloud supplies to the FS confidential identity material for the new thing which allows the FS to establish initial credentials of the thing on the blockchain. It also shares with the thing a symmetric key. Also note that a modern IoT device is equipped with hardware encryption facilities and can hold the key in software-unreadable, immutable persistent memory inside its Hardware Security Module (HSM).

- as part of the previous, supplying to a thing that joins the blockchain late the authenticated hash of the relevant blockchain history.

- withdrawal of a thing from the blockchain.

The FS is the actor that forms blocks for the blockchain by gathering and validating SLVP protocol messages (see the section "Posting on the blockchain") from things and outside agents (via Cloud) and collecting them into blocks. No trust is required for this, since the FS processes messages received from things and agents on the basis of the blockchain content, which is public and available to all users. Any violation of the validation rules will be noticed by the parties affected and any well equipped witness, i.e. a (possibly non-enrolled) radio listening post.

\footnotetext{
${ }^{2}$ Whether the increased vulnerability to a pre-image attack is significant depends on the application's specific threat model. In most cases it is not, since the attacker would only have a few minutes between the consecutive blocks for the attack. For the avoidance of doubt, we do not demand the hash size reduction, only suggesting it for resource optimisation. As a matter of fact, our solution would accommodate a double-length hash, 512 bits, just as easily should it become necessary.
}

What cannot be protected $100 \%$ reliably by our methods is progress. It is possible in principle for the FS to deny service by refusing to react to valid, legitimate messages sent by things, or by maliciously modifying the content of those messages, if the FS is compromised. However, server protection is not an issue of IoT security, but a general cybersecurity concern, which is beyond the scope of this paper.

Proxies This is yet another kind of zero-trust agent. It has two functions:

1. Amplify the Sequencer's radio broadcasts (by constructive interference, if LoRa communications are used, or by re-broadcasting broadcast messages). All Proxies are sent PLS messages by the Sequencer via the FS just before they are due to be broadcast.

2. Pick up messages from things directed to the blockchain. This is useful because direct connection to the FS over the air may not be possible given a low power budget and compromise antennae that things have to work with. All Proxies are connected to the intranet and can forward messages to the server and CAS. The blockchain posting protocol (SLVP), which we will introduce in the next section, is robust: Proxies can forward messages of unclear origin and authority; at best they will be filtered out by the FS, at worst they will find their way to the blockchain but will not be properly signed by a legitimate actor and hence will have no effect other than waste of resources.

Since all Proxies are connected to the intranet, they can perform function 1 above.

Communications The reader will have noticed that the proposed architecture uses the combination of a dedicated radio channel for security-related data and a general-purpose communication infrastructure, whether wired or wireless, on which things may establish an auxiliary channel. This is a deliberate choice for the following reasons.

The purpose of the dedicated radio channel is to ensure by physical means the reliability of broadcast. The only credible threat to the PLS protocol is a DoS attack whereby one or more users of the network are prevented from getting uncorrupted P, L or S messages sent by the Sequencer. The architecture enables a volume transmission of these messages, with the Sequencer radiating them at maximum legal power and the Proxies joining in by concurrent transmission. We propose that the security radio channel is implemented via LoRa (Sentech Corporation 2019), a Long Range spread-spectrum technology with many remarkable properties. 
We exploit the fact that as a spread spectrum format, LoRa benefits from concurrent transmissions, where two or more signals carrying the same content under the same modulation regime can be broadcast simultaneously from different locations. Any receiver at whose location the signal of one transmitter exceeds the rest only by a factor of 2 (3dB) will not sense the others ((Liao et al. 2017) p.21436, under "Capture Effect", (Zhu et al. 2018)). This is due to the Frequency Modulated (chirp) nature of LoRa, and is well known as the capture effect in both communication and FM broadcast industry (Leentvaar and Flint 1976).

The idea is to endow the Proxies with maximum legal power transmission capabilities and to place them in such a way that the communication range of each Proxy captures a certain structural unit on the premises, e.g. a floor (or a building if it is small enough), to form a communication locus. Different loci are separated by distances (as in the case of loci as individual buildings) or obstacles (e.g. construction elements supporting a floor if the loci belong to different floors). This was studied at length in (Liao et al. 2017) (see p.21443 under "The Robustness of CT-LoRa"). The distance is by itself quite an effective dampener, as radio signals fade by $6 \mathrm{db}$ when the distance between transmitter and receiver is doubled, which would ensure a more than sufficient power contrast for the capture effect.

If properly deployed, the proposed architecture ensures that an attacker can only deny LoRa communications to an IoT node by radiating power far exceeding legal limits, or by installing additional equipment on the premises in violation of physical security. In both cases a large proportion of the IoT devices will remain unaffected by the attack. They will be able to collect all versions of the security messages (including genuine ones and those coming from the attacker) and exchange them between each other using an unprotected network. Security protocols will then quickly establish which versions are genuine.

\section{Posting on the blockchain}

It is tempting to use a protocol similar to what we described in the section "Basic protocols" in order for an IoT device to post transactions directly on the blockchain. The advantage of a Guy Fawkes type protocol (which we will call a GF protocol for short) is that any secrets are short-lived, the calculations basic and post-quantum, and communications modest. However the principle vulnerability of such a protocol is its inherent reliance on the precedence of events. With PLS we used wall-clock time to separate intervals; wall-clock time with a reasonable accuracy (less than tens of seconds drift over a year) is cheap and available to even the tiniest of IoT platforms. However, a thing does not generally need to post a transaction on each block of the blockchain, and it is often quite expensive for it to do so from the energy perspec- tive. Precedence can easily be established if the protocol publishes messages on the ledger using an ideal communication environment, where all messages reach their destination and all can be published in the interval that they were emitted. Which is far from reality in the IoT world.

Jam-spoof attack. With Sequencer broadcasting messages for all users without exception, having maximum legal transmission power and being further supported by Proxies, one can guarantee message delivery (possibly several unauthenticated versions, but that is no problem) either directly, or via subsequent exchanges between users before the interval is over. When a thing sends its content to the FS, the content is of no interest to the peers and the resources available for delivering it are quite limited. If the thing posts on the chain infrequently, at unpredictable times, any GF protocol based on imperfect communication is potentially vulnerable to the jam-spoof attack (see the section "Original GFP") as follows:

(below $T$ is a thing and $M$ is an attacker)

1. T runs the protocol to the point where it is about to reveal to a verifier the so far confidential pre-image to prove a signature (a key feature of any GF protocol)

2. M suppresses the verifier's receiver by jamming the broadcast channel. At the same time $M$ uses a highgain directional antenna and sophisticated signal reconstruction techniques beyond the capabilities of the verifier to reliably receive the message from $T$. As a result $M$ learns the secret, but the verifier is left believing that the secret has not been revealed yet.

3. $M$ masquerading as $T$ proceeds to publish its linkand proof-records based on the knowledge of the secret pre-image. The link-message will use a different next nonce than the genuine link-message from $T$ published on the blockchain earlier, but the same current nonce, thus forking T's GF sequence. Also the knowledge of both nonces enables $M$ to post its own signature message on the blockchain to sign an arbitrary hash on behalf of $T$ and to continue to do so indefinitely.

4. T sees the split of its sequence on the blockchain and alerts the FS, but now the FS (or any other arbitrator) is unable, based solely on the blockchain content, to determine whether it is $T$ or $M$ that is the genuine originator of the latest messages.

Two remedies are available. One is to require authentication of all messages from a thing to the FS. This immediately destroys the zero trust environment we have built, where the only aspect that all users, including the FS itself, have to trust is the correct operation of the Sequencer. We wish to implement a GF protocol that does not require additional trust and which survives a jam-spoof attack. It 
Table 2 SLVP Protocol

\begin{tabular}{|c|c|c|c|}
\hline Block & Transmit & Verification & BC Action \\
\hline$i_{0}$ & $P_{1}=H\left(N_{1}\right)$ & Out of Band (Enrolment) & \\
\hline$i_{1}$ & $S_{1}=\mathbf{E}_{N_{1}}\left(H\left(M_{1}\right) \oplus H\left(N_{2}\right)\right)$ & & \\
\hline$i_{2}$ & $L_{1}\left\|V_{1}=H\left(N_{2}\right) \oplus N_{1}\right\| H\left(H\left(N_{2}\right) \| N_{1}\right)$ & & \\
\hline$\ldots$ & $\ldots$ & $\ldots$ & $\ldots$ \\
\hline$i_{n}$ & $P_{k}=H\left(N_{k}\right)$ & $\ldots$ & post $P_{k}$ \\
\hline$i_{n+1}$ & $S_{k}=\mathbf{E}_{N_{k}}\left(H\left(M_{k}\right) \oplus H\left(N_{k+1}\right)\right)$ & & \\
\hline$i_{n+2}$ & $L_{k}\left\|V_{k}=H\left(N_{k+1}\right) \oplus N_{k}\right\| H\left(H\left(N_{k+1}\right) \| N_{k}\right)$ & & \\
\hline \multirow[t]{14}{*}{$i_{n+3}$} & $P_{k+1}=H\left(N_{k+1}\right)$ & Fetch latest $P=P_{k}$ from block $B=i_{n}$ & \\
\hline & & Set failed=true & \\
\hline & & for $L V: B<\#(L V)<i_{n+3}$ & \\
\hline & & if $H\left(L \oplus P_{k+1}\right) \neq P$, continue & \\
\hline & & $\operatorname{set} N=L \oplus P_{k+1}$ & \\
\hline & & if $H\left(P_{k+1} \| N\right)=V$ & \\
\hline & & failed=false; break & \\
\hline & & if failed, exit & ignore $P_{k+1}$ \\
\hline & & for $L^{\prime} V^{\prime}: B<\#\left(L^{\prime} V^{\prime}\right)<\#(L V)$ & \\
\hline & & if $V^{\prime}=H\left(L^{\prime} \oplus N \| N\right)$, exit & ignore $P_{k+1}$ \\
\hline & & for all $S: B<\#(S)<\#(L V)$ & \\
\hline & & determine $H_{M}=\mathbf{D}_{N} S \oplus P_{k+1}$ & \\
\hline & & send to CAS: $\left(k, \cup I D, H_{M}, \alpha(L V), \alpha(S)\right)$ & post $P_{k+1}$ \\
\hline & & exit & \\
\hline
\end{tabular}

Assume bitwise exclusive-or $\oplus$ to have a higher priority than concatenation $\| . \#(x)$ is the number of the block in which record $x$ is located, and $\alpha(x)$ is the record's unique ID (address). The sequence of block numbers is in strictly increasing order: $i_{0}<i_{1}<i_{2}<\ldots$

turns out that a small modification is sufficient to solve the problem, which brings us to the following SLVP protocol, see Table 2 .

\section{SLVP protocol}

A new blockchain user is enrolled by the Fog Server ${ }^{3}$ by authenticating the user's first P-record out of band. Assume that the user sends that record to the blockchain (i.e. to the FS in the first place) and it appears in some block $i_{0}$.

All records on the blockchain carry a UID, i.e. the User IDentification, stated by the message originator. We propose that the user is identified by the first two bytes of its $P_{1}$ record. The server will not accept the $P_{1}$ message if it determines that the first two bytes of the hash clash with an already established user. On the other hand, 2 bytes are sufficient for an excess of 64 thousand users, while a typical IoT swarm does not exceed ${ }^{4} 1000$. In the sequel we will not distinguish between a UID and the user with that UID, if the context is clear enough to see which of the two we mean.

\footnotetext{
${ }^{3}$ this makes it a permissioned blockchain

${ }^{4}$ There is some evidence that 1000 nodes could saturate the IoT long-range joint channel capacity (Haxhibeqiri et al. 2017)
}

The table in Table 2 presents the protocol from the point of view of a single UID and the FS. Other UIDs will conduct themselves in the same way. Like the PLS protocol introduced earlier, the SLVP protocol is invoked periodically, but now at some arbitrary times, which in terms of the blockchain schedule correspond to block intervals. The transmitting thing does not need a specific confirmation that the message has been received; it simply checks newly formed blocks to find the record that it has transmitted. When this happens, the thing sends the next message according to the protocol. If a legitimate transmitter's correct message is not posted on the blockchain after it has been transmitted, it either wasn't received at all, or it was received distorted. Either way the thing will re-send the message until it is posted on the blockchain intact.

The protocol proceeds in rounds, each consisting in three steps:

$$
S \rightarrow L V \rightarrow P
$$

where $S$ is, as before, the signature record, $L V$ is an extended link-verify record, and $P$ is the proof record as before. The $L V$ record consists of the $L$-record, similar to that of the PLS protocol, and a $V$-record for thwarting 
the jam-spoof attack. Since the UID is not authenticated and the channel generally lacks integrity, any messages received by the blockchain can be arbitrarily distorted.

The SLVP protocol depends on things' ability to receive blocks on the blockchain successfully via the PLS protocol. Unlike the Sequencer engaging in timed broadcasts, an SLVP user can be quiescent for many block periods. The new round $k$ starts after the user's $P_{k}$ message has been received by the FS, validated and posted on the blockchain in some block $i_{n}$. Which block this is going to be depends on the timing of the $P_{k}$ message. The user, having satisfied itself that its message $P_{k}$ was received and posted under its UID, sends the $S$-message $S_{k}$ and waits for it to get posted, too, say in block $i_{n+1}>i_{n}$.

Having satisfied itself that the $S_{k}$ has been posted on the blockchain, the user transmits its $L V$-message.

The message $P_{k+1}$ is sent in some later block period $i_{n+3}$ to finish the current round. The FS verifies the message using the verification procedure in the middle column of the table in Table 2 . If verification succeeds, the new $P$ record is posted.

Next the FS will decipher all $S$-records posted after block $i_{n}$ under the user's UID by computing

$$
H_{M}=P_{k+1} \oplus \mathbf{D}_{P_{k+1} \oplus L} S .
$$

Each record $r$ on the blockchain has its address $\alpha(r)=$ $\left(i_{r}, l_{r}\right)$, where $i_{r}$ is the block number in which $r$ is located and $l_{r}$ is the sequential number of $r$ among the records of the same type and under the same UID in block $i_{r}$.

For each record $S$ in the current round $k$, the FS collects proof data in the following form:

$$
W_{S}=\left(k, \mathrm{UID}, H_{M}, \alpha(L V), \alpha(S)\right)
$$

and instructs the CAS unit to store $W_{S}$ under $H\left(W_{S}\right)$ as usual. The CAS unit will use $H_{M}$ as a trigger. When/if the user UID stores content $c$ in CAS, such that $H(c)=H_{M}$, the CAS manager will post a special $C$-record, on the blockchain on behalf of UID as follows:

$$
C=\mathrm{UID}:\left(H_{M}, H\left(W_{S}\right)\right)
$$

which serves as blockchain confirmation that CAS has taken charge of the content file as well as the proof data for it for any witness to verify. Triggers that are not triggered by the user over a certain number of blocks (large enough to conclude that the original $S$-message was counterfeit/distorted) are removed from CAS and entered into the FS security log. A $C$-message will be ignored (and the corresponding $C$-record not posted) if a trigger for it has not been provided by the FS at the time of submission.

\section{Security analysis of SLVP}

An $S$-message does not expose a single bit of the nonces $N_{k}$ and $N_{k+1}$ since the value $S_{k}$ depends on yet undisclosed $H\left(M_{k}\right)$ and since there does not exist an attack on the cipher $\mathbf{E}$ where neither the key nor the plaintext is known. Sometimes it is convenient for the user to post more than one $S$-record, for example when several documents are to be signed by the same user but they are not otherwise related. The user is allowed to send as many different $S$-messages as necessary.

For an $L V$-message, the link part, $L$ is the same as that in PLS, and it serves the same purpose: its value links the current nonce $N_{k}$ with the new one, $N_{k+1}$. The verify part $V$ is there to make sure that an attacker who learns $N_{k}$ later cannot combine it with its own $\hat{N}_{k+1}$ and post

$$
\hat{L}_{k}=H\left(\hat{N}_{k+1}\right) \oplus N_{k}
$$

on the blockchain. In such a case the FS would be unable to decide between $L_{k}$ and $\hat{L}_{k}$ due to the fact that $L_{k}$ can be a distorted version of $\hat{L}_{k}$, and the message $\hat{L}_{k}$ an attempt to correct the distortion. With the $V$ message in place, for any pair of $L V$-records:

$$
L_{k}\left\|V_{k}=H\left(N_{k+1}\right) \oplus N_{k}\right\| H\left(H\left(N_{k+1}\right) \| N_{k}\right)
$$

and

$$
\hat{L}_{k}\left\|\hat{V}_{k}=H\left(\hat{N}_{k+1}\right) \oplus N_{k}\right\| H\left(H\left(\hat{N}_{k+1}\right) \| N_{k}\right),
$$

where

$$
H\left(L_{k} \oplus P_{k+1}\right)=H\left(\hat{L}_{k} \oplus P_{k+1}\right)=P_{k},
$$

the one posted in an earlier block wins: the protocolcompliant user does not disclose the genuine $P_{k+1}=$ $H\left(N_{k+1}\right)$ in the same block as $L_{k} \| V_{k}$ and so the fact that

$$
V_{k}=H\left(P_{k+1}|| L_{k} \oplus P_{k+1}\right)
$$

proves that the originator knew $H\left(N_{k+1}\right)$ before it was posted. The only actor that knows $H\left(N_{k+1}\right)$ before it is posted is the genuine user.

Notice that no matter how many counterfeit $L V$ messages have been posted by attackers since the last verified $P$ and no matter how many counterfeit $P$-records are sent after them, only one $P$ record will be accepted and posted by the FS in any round of the protocol on behalf of any given UID. Also the FS will find only one $L V$-record to be valid, which is the earliest $L V$-record compatible with both the previous and the newly validated $P$-record.

Counterfeit $S$-messages pose no threat. They will be deciphered to an unpredictable $H_{M}$, and an attacker would not be able to provide content that matches a given hash value anymore than it is able to find a nonce $N$ given $H(N)$, the latter being the main security assumption for any GF protocol. 


\section{Enrolment and optimisations}

It has been mentioned earlier that the very first hash $P_{1}$ of the protocols is validated out of band. For a user to be able to start SLVP there are two requirements:

- access to the blockchain which includes out of band validation of the latest $P_{k}$ and all previous blocks from 1 to $k-1$, inclusively

- registration of the user's $P_{1}$ for out of band validation.

Enrolment of new equipment normally requires a human administrator as it involves physical placement, configuration and initialisation of the item according to the business objectives. We propose the following enrolment protocol:

1. The administrator's workstation establishes secure confidential communication with the FS using Cloud and state of the art security. The FS shares a fresh key $K$ with the administrator.

2. The new thing that the administrator has ascertained to be genuine

(a) receives $K$ and the Sequencer's latest $P_{k}$ using near-field communications (NFC) or similar,

(b) generates $N_{1}$ and another random nonce $N^{*}$

(c) computes $P_{1}=H\left(N_{1}\right)$

(d) sends $Q=P_{1} \| \mathbf{E}_{K}\left(P_{1} \oplus N^{*}\right)$ back to the server via the administrator's NFC port acting as a relay.

3. The FS examines a short prefix of $P_{1}, \pi\left(P_{1}\right)$, e.g. 2 bytes, and checks that no UID with this value has been enrolled. If that is the case, the server computes $N^{*}$ from Q and responds with ACK $=H\left(N^{*}\right)$ otherwise the response is FAIL.

4. If the response is FAIL, the thing generates a new pair $N_{1}$ and $N^{*}$ and repeats steps 2 and 3. Otherwise

(a) the thing verifies that $\mathrm{ACK}=H\left(N^{*}\right)$ and notes its new UID, i.e. $\pi\left(P_{1}\right)$

(b) confirms completion to the administrator.

If $\mathrm{ACK} \neq H\left(N^{*}\right)$ the protocol fails; a notification to this effect quoting $P_{1}, N^{*}$ and ACK is sent to the administrator for subsequent analysis.

Now the new IoT device is ready to receive the minimum data necessary to access the blockchain. The amount of trust required for it is exactly the same as it is for any other user: it needs to authenticate the latest $P_{k}$, the only difference being that for the devices that have been present from the start the index $k=1$. But how is it going to authenticate the blocks that were formed before block $k$ ?

Merkle tree. To describe our proposed solution we need to remind the reader the idea behind the Merkle tree first.

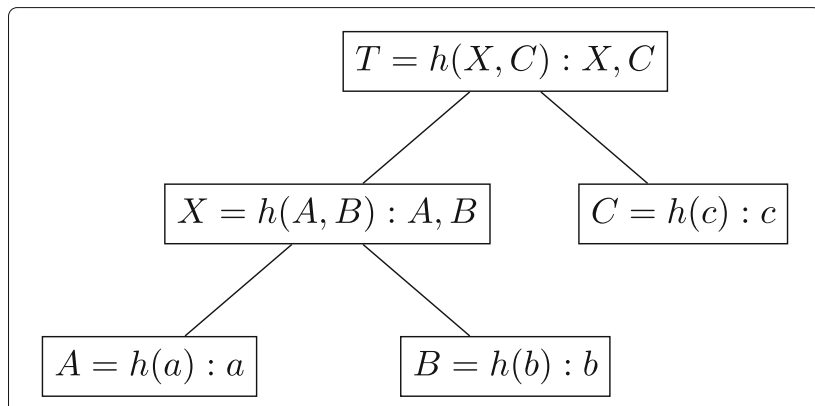

Fig. 2 An example of the Merkele tree

The Merkle tree is a tree in which every leaf node is labelled with the cryptographic hash of a data block, and every non-leaf node is labelled with the cryptographic hash of the labels of its child nodes. See Fig. 2 for an example.

Based on the same fundamental hardness of finding a second pre-image, it can be concluded that if the top-level label is authenticated reliably, then no further node of the tree needs to be. Indeed, it is cryptographically hard to change, for example, the data block $a$ without changing the label $A$, and if $A$ changes, the value of $X$ and consequently $T$ will become invalid. So one hash value is sufficient to authenticate the whole data structure. At first glance, the tree seems to be an unnecessary complication, since the hash of $a, b$, and $c$ alone, $h(a, b, c)$ would be sufficient to assure the integrity of all three items. However, the advantage of the Merkle tree is that it allows one to access just the items that one wants. For instance, to access $a$, one does not have to read $b$ and $c$; all that's required is to read nodes $T, X$ and $A$. The amount of data retrieved will be $|a|+5|h|$, where the vertical bars denote the item $\operatorname{size}^{5}$. Without the Merkle tree, in the case of a single hash controlling $a, b$ and $c$, the retriever would have to read $b$ and $c$ and compute the hash to assure the integrity of $a$, even when the contents of data blocks $b$ and $c$ are of no interest. Since items can be of an unlimited size and the hash size is limited and small, the Merkle tree offers a clear advantage in both communication and hash calculation costs. Now let us proceed to our proposed data structure.

Merkle forest. We propose to communicate the Merkle minimal forest roots of the current state in each blockchain block. The roots are a compact collection of root hashes that can be followed on CAS to securely access any block from $B_{1}$ to current stored in CAS. To illustrate the concept, let us imagine a block sequence from block 1 (initial) to block 7 (current), see Fig. 3. For simplicity we use a binary Merkle tree, in which every non-leaf node

\footnotetext{
5 the label of a node is not stored at the node (except for the top node). The retriever will read $T, X, C$ (and verify that $T=h(X, C)$ ) from the top node, $A$ and $B$ from node $X$ and finally a from node $A$, which works out as 5 hash lengths plus the length of $a$.
} 
is composed of the hashes of the two child nodes' contents. Some of the nodes are already formed and will never change (shaded in the figure), and some are still being formed pending the future blocks. It is easy to see that the Merkle proof of any block up to and including 7 requires only hashes of node 4321,65 , and 7 as roots, the paths of every leaf from 1 to 7 is rooted at one of them. Notice that the binary representation of 7 is 111 which corresponds to one node each at levels 0,1 and 2 . For block $5=101_{2}$ we would have a level-2 block and a level-0 block, which agrees with the diagram.

A binary Merkle tree is unjustifiably deep. Focusing on the world of the IoT, we recognise that communications are typically limited to messages no longer than 200-250 bytes, so given a typical hash size of 32 bytes, it is convenient to use a quad-tree, which will be much less deep. For a quad tree in a blockchain of say, 1 mln blocks (at one block per $15 \mathrm{~min}$, this gives us more than 10 years' running), we get $\log _{4} 10^{6} \approx 10$, which means that the server only needs to authenticate at most 10 hashes to give a new IoT thing a secure start. For every $k$ the record consisting of the minimal root set

$$
\Gamma(k)=\left(r_{1}, \ldots, r_{p}\right)
$$

is computed and stored by the FS under $\gamma_{k}=H(\Gamma(k))$ in CAS, where $k$ is the current block number, and $r_{1}, \ldots, r_{p}$ are the Merkle tree hashes that correspond to the nonzero digits in the base-4 representation of the number $k$.

For each $k$, the FS will put $\gamma_{k}$ at the beginning of block $k+1$. Any user that wishes to trade storage for CAS communication, or a new user who has missed an initial segment of the block chain, but who trusts the latest $P_{k}$ can use the $\gamma$-record on the block to securely retrieve any preceding block(s) via CAS, if they choose to trust the FS. We would like to remark that the FS in this particular case is trustworthy, since the $\gamma$ record can be computed by any full witness of the blockchain (i.e. any user that has been present since block 1) and if the FS is compromised, the proof of that will be constructed immediately.

Regarding the storage requirement in CAS, they are minimal. Summing up the geometric series for the radix4 Merkle tree with depth 10, we get circa 350,000 hashes to store for $10^{6}$ blocks, about $10 \mathrm{Mb}$, a trivial amount of storage.

\section{Countermeasures against DoS}

The acquisition of a shared secret between the thing and the FS at the point of enrolment does not make the blockchain any less useful. Indeed, in our threat model the FS is not trusted by any enrolled user any more than any other user of the blockchain, so the shared secret cannot be used to replace the security protocols that make the blocks of the blockchain an immutable, ordered, authenticated sequence of records. Nor is it any good for non-repudiation. However, just as the Sequencer is trusted to keep its secret for the avoidance of blockchain split so is the FS trusted to be interested in reducing the amount of noise on the blockchain, i.e. records sent in by an attacker on behalf of a genuine UID, which will eventually be caught out and eliminated by the SLVP protocol. After all, as the FS is solely responsible for what does and what does not get posted, the proposed blockchain concept only works on the assumption that the FS itself is not and can never be behind a DoS attack. The assumption that the FS will be a willing party to an additional noise-reduction protocol does not add much to that.

With this in mind we propose that each thing uses a very short Message Authentication Code (MAC) based on symmetric encryption and the shared key $K$ received upon enrolment. The MAC need not be longer than

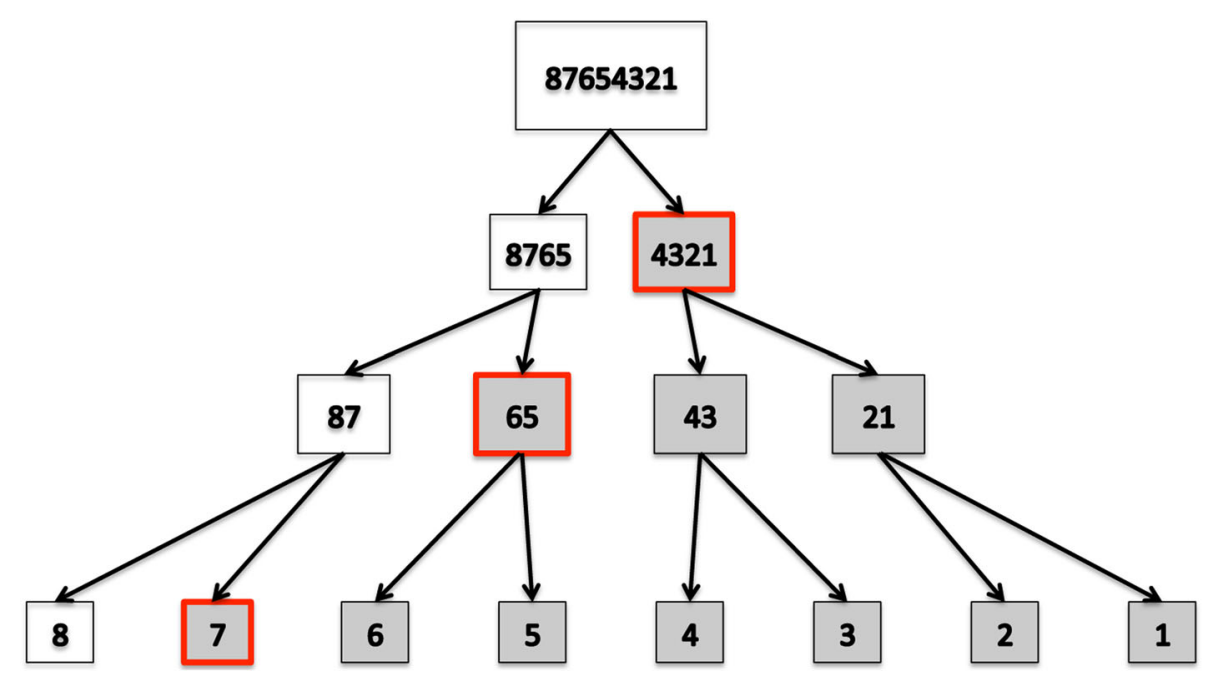

Fig. 3 Merkle forest 
2 bytes (possibly even 1 byte) and can be computed using standard techniques by the thing's hardware security module or crypto accelerator. The MAC is computed for each message of the SLVP protocol sent to the server as well as the content messages sent via the FS to CAS. Due to the shortness of the MAC, the exposure of the shared key is minimal, obviating session keys. If the MAC does not match, the FS ignores the message. With a 2-byte $\mathrm{MAC}$, an attacker would have to send tens of thousands of messages to get through to the FS in the first instance; such a volume on behalf of a single IoT user will surely raise the alarm, resulting in the intruder's triangulation and suppression.

Recall that the Sequencer's messages may arrive distorted or not arrive at all, and the users, especially things, must talk to each other to collect a set of versions for each PLS message to ensure that the set contains the original. To facilitate this, a short authenticator can be sent by a Proxy on an auxiliary channel to each thing by transmitting

$$
u=\mathrm{UID}\|\mathbf{c a t}\| \pi(H(M))
$$

where UID is its User ID, $\pi(H(M))$ is a short hash of message $M$ from the category cat (one of $P, L$, or $S$ ). Message $u$ is extended with $\operatorname{MAC}_{K}(u)$, where $K$ is the key agreed with UID at enrolment. The message $u$ is prepared by the server and is forwarded by one or more of the Proxies on the auxiliary channel. User UID, having received $u$ and checked the MAC, recalculates $\pi(H(M))$ based on the latest message in category cat received (if it did at all) and compares it with the value contained in $u$. If they match, the device joins a re-broadcast concurrent-transmission group on a pre-arranged channel (frequency and time relative to the start of the Sequencer broadcast interval) to help nearby nodes with PLS reception. Given that PLS messages are short (not much longer than 32 bytes if SHA256 is used for $H(\cdot)$ ) and infrequent ( 3 messages typically 2-5 times per hour, $0.5 \mathrm{~KB} /$ hour), a blockchain supported thing can afford to transmit as much to help other things (which in turn will help it) to survive a DoS attack.

When it comes to the SLVP protocol, the user is the active transmitter, and the roles are reversed. Now as a DoS resilience measure, the user UID adds a $\operatorname{MAC}_{K}(x)$ to every message $x$ that it sends to the server (possibly via a Proxy) for posting on the blockchain. The FS checks the MAC based on the received UID and the shared key $K$ and if the MAC does not match, it ignores $x$. Again, we must stress that if the MAC does match, this means nothing in terms of the SLVP protocol, since the FS does not trust the thing any more than the thing trusts the FS. Reduction of noise is their common concern: the FS acts on behalf of the owner of the IoT network and is interested in suppression of a DoS attacker, and the thing will keep its shared $K$ secret to avoid an attacker's spoofing it and preventing its legitimate messages from reaching the blockchain. Commonality of concern is the only reason why the additional authentication will be effective.

\section{Emergency mode}

A distinguishing feature of IoT is its multiplicity of time scales. Most things require only infrequent interaction with the outside world, reporting sensor readings, receiving parameter updates and possibly code upgrades. All these activities are easily accommodated by the blockchain mechanism and are protected by its inherent security properties. A major downside of a blockchain is its latency. No matter how frequently new blocks are added to the chain (and in our case they are not even mined), a thing may find itself in a situation when it must raise the alarm with its master sooner than a new block can be published, especially since in the case of the PLS blockchain, blocks are published on a fixed wall-clock schedule. Even if a new block is to emerge soon, there is no guarantee that any given thing will be able to post its message in it rather than in a later block.

This problem is quite practical: a hospital monitor detecting a catastrophic change in a patient's condition and a nuclear plant's sensor detecting a reactor malfunctioning are cases in point to name but two. We emphasise that emergency messages are not an alternative of posting records on the blockchain. The latter is more powerful in that things are able to securely interact with each other directly via their signed blockchain messages, whereas emergency communications are processed solely at the FS for off-chain delivery outside the IoT network.

One might think that emergency communications can be supported by the shared key $K$ that the originating thing agreed at enrolment. Indeed the FS can request a full MAC and satisfy itself that the message is authentic. However, this is not enough. Emergency communications involve rapid response and that can only be provided if an independent arbitrator can establish that the message was sent by no-one but the claimed originator. In other words, a signature rather than mere authentication is required. In the absence of signature, the response agent would be running the risk of the originator repudiating the message: after all, the symmetric key $K$ is shared with the FS, and so either the FS or an agent to which the FS has leaked the key (willingly or not) might have sent the emergency message instead of the legitimate user.

Non-repudiation is not a concern with blockchain communications, they cannot be repudiated thanks to the properties of the SLVP protocol. However, post hoc validation by blockchain is only useful for confirmation of valid messages, rather than proving a message to be invalid, since the rapid response must come into effect before blockchain validation may take place. The other 
way $^{6}$ of ensuring non-repudiation is by One-Time Signature (OTS), which we will consider next.

\section{OTS}

OTSs are known to have a very large "public key", i.e. authenticated public data used for validation of a signature. In the original OTS proposed by Lamport (Lamport 1979), the originator shares with the verifier $k$ pairs

$$
\left(H\left(n_{1}\right), H\left(N_{1}\right)\right),\left(H\left(n_{2}\right), H\left(N_{2}\right)\right), \ldots,\left(H\left(n_{k}\right), H\left(N_{k}\right)\right),
$$

where all $n_{i}, N_{i}$ are random nonces. To sign a $k$-bit message $\left\{x_{i}\right\}$, the originator additionally supplies $k$ values $\left\{s_{i}\right\}$ :

$$
s_{i}= \begin{cases}n_{i} & \text { if } x_{i}=0 \\ N_{i} \text { otherwise }\end{cases}
$$

OTSs solve the problem of emergency non-repudiation if the public key is signed and posted on the blockchain in advance (using SLVP), but the price for an IoT device using it is prohibitive. A straightforward application of OTS to signing a full hash of an emergency message would require $256 \times 256 \times 2=128 \mathrm{~K}$ bits of public key, or $16 \mathrm{~KB}$. It is easy to see that the public key can only be used once if we want the security of hash pre-image to work for every bit of a signed message. Even if using the large key once were acceptable (think of catastrophic circumstances that do not present themselves often), the signature size would also be prohibitive: half the key size, or $8 \mathrm{~KB}$ in this case. This would take some time to communicate over a low bit-rate channel, especially in the presence of transmission errors necessitating a re-transmission.

\section{Public key}

Let us start with the public key problem. We propose to bring the SLVP protocol messages to bear on the emergency mode to eliminate transmission and authentication of the public key. Recall that a thing running the protocol sends messages that depend on nonces $N_{k}$ which are chosen by it at random, see Table 2. At the validation step, the FS computes $\hat{N}_{k}=L_{k} \oplus P_{k+1}$ and verifies that $\hat{N}_{k}=N_{k}$ by applying $H(\cdot)$ to both sides and checking the equality.

We propose that every thing engaging in SLVP must compute random nonces $N_{k}$ by building a hash chain:

$$
\begin{aligned}
N_{k} & =N_{k}^{[\alpha]} \\
N_{k}^{[i]} & =H\left(N_{k}^{[i-1]}\right) \text { where } i=1, \ldots, \alpha,
\end{aligned}
$$

and where $N_{k}^{[0]}$ is completely random and is kept secret by the thing for at least $\alpha$ rounds of the protocol.

In other words, every nonce is an image of a random number under $\alpha$ applications of $H(\cdot)$, which is known as the Winternitz chain. When the server has posted the

\footnotetext{
${ }^{6}$ Our design constraint 1, Post Quantum, prevents standard public-key cryptography, which would provide an effective signature if the originator's public key is validated in advance on the blockchain
}

value $P_{k}$ it received from the thing, it has access to, and has verified,

$$
N_{i}^{[\alpha]} \text { for all } i=0, \ldots, k-1
$$

For a given UID, the private key for the period between the postings of $P_{k}$ and $P_{k+1}$ (i.e. when nonces up to and including $N_{k-1}$ have been revealed) is as follows:

$$
\left\{N_{k-i}^{[\alpha-i-1]}\right\} \text { for } i=1, \ldots, \alpha-1 .
$$

If Lamport's OTS is used, the thing sends to the FS a selection of the values $N_{k-i}^{[\alpha-i-1]}$. The FS verifies each value by applying $H(\cdot)$ to it $i+1$ times and comparing the result with $N_{k-i}^{[\alpha]}=N_{k-i}$ that it has received from the same UID by SLVP protocol. Notice that as $k$ advances to $k+1$ with a new round of SLVP, the same chain $N_{k-i}$ is used for public key with an earlier pre-image:

$$
N_{k-i}^{[\alpha-i]} \rightarrow N_{(k+1)-(i+1)}^{[\alpha-(i+1)]}
$$

until the protocol is $\alpha$ rounds ahead of the chain at which point the chain will have been fully used and is no longer required for OTS purposes. An example of $\alpha=3$ is displayed in Fig. 4, showing two consecutive rounds. Notice that the values used in a later round are always lower on their Winternitz chain than those in earlier rounds, making them secure within the second pre-image hardness assumption.

We conclude that the "private key" for an SLVP round, i.e. the set of potential pre-images to be used for OTS, is unknown to the FS in that round and that the FS has access to the authenticated public key to verify the signature.

This arrangement of chains and pre-images makes it possible for a thing running SLVP not to share any public key at all and at the same time be able to sign messages in emergency mode without waiting for blocks to appear on the blockchain. It is quite useful for the IoT world, and the

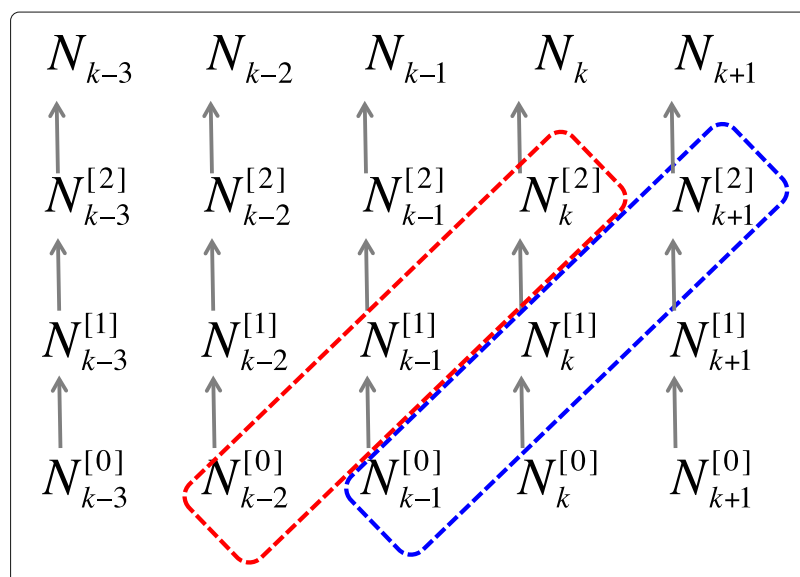

Fig. 4 Use of Winternitz chains for OTS private key production from SLVP nonces at round $k$ (red) and $k+1$ (blue) for $\alpha=3$. NB: $N_{i}^{[3]}=N_{i}$ so the top row is shown without superscripts. Vertical arrows signify application of $H(\cdot)$ 
price that we pay is the need to pre-hash a random string $\alpha$ times at every round of the SLVP protocol rather than use it directly as a nonce. Taking a popular ESP32 systemon-chip as a specific example we learn from (Espressif Systems 2020) that it takes $\sim 1 \mu$ s at full power to process one AES256 hash block, perhaps $0.1 \mathrm{~ms}$ for $\alpha=100$. The energy spent is a fraction of the (LoRa) communication cost for the same. It is completely justified if the thing potentially requires emergency communications in this or any of the future $\alpha$ rounds, assuming that it is sufficient to sign a certain number of bits $L$ of the emergency message (or its digest) to reassure the responder of nonrepudiation. For Lamport's classical OTS signature, $L=\alpha / 2$. We will improve on this next.

\section{GF-HORS}

The excessive size of OTS signatures have been recognised by many authors, and several proposals have been made to improve on it. We follow the methodology presented in (Reyzin and Reyzin 2002), where an original idea, Hash to Obtain a Random Subset (HORS), was first put forward.

Assume that $\alpha$ is a power of 2. Compute a length- $L$ digest of the message to be signed, and partition its binary representation into slices $\log _{2} \alpha$ bits long. Interpret these slices as unsigned numbers

$$
\sigma_{j}, j=0, \ldots, \frac{L}{\log _{2} \alpha}-1 .
$$

where all $\sigma_{j}<\alpha$. Now for each $j$ the thing supplies the value $N_{k-\sigma_{j}}^{\left[\alpha-\sigma_{j}-1\right]}$ to form a signature. The FS validates the signature by recomputing the digest of the message, then recomputing $\left\{\sigma_{j}\right\}$ from the digest, and then for each $j$ verifying $N_{k-\sigma_{j}}^{\left[\alpha-\sigma_{j}-1\right]}$ by applying $H(\cdot)$ to it $\sigma_{j}+1$ times and checking that the result equals the previously obtained nonce $N_{k-\sigma_{j}}$.

The idea to use the digest of the message to be signed rather than the actual bits of it by partitioning the string was first proposed in (Reyzin and Reyzin 2002), and the security of this method is slightly less than that of the second pre-image hardness, since here the attacker only needs to find a message whose digest partitioned into suitable chunks gives the same set or even a subset of $\left\{\sigma_{j}\right\}$ in any order. However, the authors of (Reyzin and Reyzin 2002) remark that finding a (useful) message that has the same set or a subset of digest chunks as a given one is still computationally hard for a good digest.

We propose a GF-HORS signature (HORS signature with SLVP-derived public key) based on a keyed MAC with the shared key $K$ as the digest. The MAC protects the message being signed from an outside forgery, and the HORS signature protects it from an insider job. Let us take a look at some example numbers to illustrate the efficiency of the scheme.
If we assume $\alpha=64$ and use AES-128 for the digest MAC (shortening it down to 126), we get up to 21 sigmas. Assuming for estimation purposes that the digest is a random bit string, the probability that an attacker's digest gives a subset of the sigmas, is less than $(21 / 64)^{21}$, around $10^{-10}$, a pretty good result for an IoT device nonrepudiation. The communication cost of the signature is $256 \times 21 / 8=672$ bytes, about three messages on LoRa. Recall that we require three shorter messages (around 128 bytes all together) for an SLVP round, which is in the same order of magnitude.

A final remark. When a thing is first enrolled by the server, there is not enough nonces in its history (in fact there aren't any initially) for the formation of the public key. One remedy could be to produce $\alpha$ nonce chains at enrolment and share $\alpha$ chain-ends with the FS at that point. Another solution is to consider the first $\alpha$ SLVP rounds of a new thing a probationary period, when it is being tested and adapted to its environment and when it is not allowed to participate in emergency communications.

\section{Related work}

The advantages of blockchain technology in the case of IoT are not clearly articulated in literature. Recent surveys (Kouicem et al. 2018), (Wang et al. 2019) recognise blockchain as a disruptive technology for the IoT, and list the benefits in generic terms:

- Decentralisation: Distributed Ledger Technology is supposed to be more robust and secure against a single point of failure.

- Pseudonymity: the ability to enrol a new actor by registering its public key (or public hash, in our case)

- Security of Transactions. This boils down to the immutability of the ledger.

This is matched with a plethora of use cases mentioned in (Kouicem et al. 2018), see pp. 212-214. However, none of the bullet points is specific for the IoT.

We find our objectives to be close to those of (Dorri et al. 2017), and that paper is a good illustration of how different our approach is from the direction inspired by the typical assumptions. The authors of (Dorri et al. 2017) assume, like others (see, for example, (Danzi et al. 2019)), that an individual IoT device is likely to be underpowered for managing blockchain transactions directly, as it does not have the storage space, communication bandwidth or processing power for such a task.

As far as communications are concerned, article (Dorri et al. 2017) correctly posits that low bit-rate radio channels, such as LoRa will be used. However it pays to differentiate between communication of a small amount of security-related data and unsecured, bulk public data transfer. 
Storage-wise, to the best of our knowledge published research assumes that the blockchain either has to be stored at the IoT device itself (which is indeed expensive), or else trust must exist between the device and any storage server. The latter assumption is not necessarily justified due to the availability of Content-Addressable Storage (CAS), which is, by construction, self-certified not requiring trust or secure communications. The idea of CAS goes back to the late 1990's paper (Crespo and Garcia-Molina 1998) where it was proposed to use a file's CRC as its name, which is not quite satisfactory due to massive aliasing, but a few years later paper (Quinlan and Dorward 2002) suggested the cryptographic hashes of files should serve as file names. In the last five years the leading general-purpose CAS project has been one known as InterPlanetary File System (IPFS) (Benet 2014) and it is widely used.

The original Guy-Fawkes protocol on which PLS is based ((Anderson et al. 1998), p.12) requires four items to be published in every round of the protocol, while PLS only publishes three. Also, verification in a round of Guy Fawkes requires a calculation that involves three items to be hashed together, whereas PLS computes a hash of one item of a minimum size, a factor of three saving on the receive side. PLS performs a symmetric decryption to obtain and confirm the message (or, to be precise, the message hash), which Guy Fawkes does not need. However, taking an example of ESP32 (Espressif Systems 2020) as a popular system-on-chip for IoT with a crypto accelerator, the AES-256 decryption calculation costs at most 22 clock cycles, while computing SHA-256 requires at least 60 clock cycles to process one block plus a minimum of 8 cycles to produce the digest. This means that a hash is at least three times as expensive as the standard encryption. This is not surprising since the security of the hash function depends solely on the diffusion properties of an iterated mapping; to achieve good diffusion as many as 80 iterations are used (64 for a shorter hash). Whereas AES encryption involves a key, which injects entropy in the process consequently reducing the need for iterations (or rounds as they are called in the area of symmetric ciphers) from the diffusion point of view: only 10 or 12 rounds are used. Modern accelerators have enough resources to perform data-independent calculations in parallel, so it is the number of strictly sequential rounds (where the input of one requires full valid output of another) that determines the speed.

We conclude that PLS is both faster and less communication-intensive at the receiver end. At the transmitter end performance matters little, since the FS and Sequencer are not on a tight energy budget.

We are aware of one prior attempt at using a GF protocol in conjunction with a blockchain: (Bonneau and Miller 2014). In that paper the blockchain itself is assumed to be Bitcoin and a GF protocol is used only for signing value transfer messages (i.e. transactions). The authors of (Bonneau and Miller 2014) were aware of the jam-spoof attack (which they call race-condition theft), but their solution is partial, based on a time-out whereas the $V$ messages in our SLVP protocol capture both pre-images, the current and the next ones, to defeat the jam-spoof attack without needing a time-out facility (but we still require the "earlier LV message wins" analysis similar to (Bonneau and Miller 2014)).

Repeated application of the hash function in order to reduce the size of the public key was first suggested by Winternitz according to Merkle (Merkle 1989). We are not aware of any prior work on our proposed sliding window across Winternitz chains. We use the original HORS (Reyzin and Reyzin 2002) procedure, but this has been improved to HORST (Bernstein et al. 2015), which we can also accommodate. HORST differs from HORS by the fact that the public key is stored in a Merkle tree, whose root is authenticated in advance (at the cost of one round of the SLVP protocol in our case). We prefer the original, HORS, as it allows us to piggy-back the public key on the SLVP nonce sequence by producing each nonce off the top of an individual Winternitz chain with the bottom kept confidential; as a result the user does not need to publish its public key at all. Not only does it save us a round of SLVP, it obviates communication of a large public key to CAS as well. However, we recognise that HORST may be useful for emergency messages if the required long-term security necessitates a much longer signature, in which case the user must store a sufficiently large HORST Merkle tree in CAS in advance and bear the risk of exposing the private key, held inside an IoT device, to a physical intruder.

\section{Conclusions}

We have presented the architecture and protocol suite for a permissioned blockchain construction based on the Guy Fawkes family of protocols. Our construction requires limited trust for one sealed, air-gapped unit we call Sequencer, which is not internet-connected and which is responsible for keeping a short-term secret. If the shortterm secret is kept, we show that this type of blockchain will not split and will maintain immutability. The rest of the network is untrusted.

We have proposed a protocol for posting signed messages on the blockchain without using public-key cryptography and discussed its security. Finally, we have shown how emergency (zero-latency) communications can coexist with a PLS blockchain without requiring a public key, yet maintaining nonrepudiation.

The main threat to the PLS blockchain is DoS attacks. While those cannot be fully eliminated for a radio network susceptible to jamming, we suggested the use of a shared secret for non-physical DoS defence: reduction of 
the number of counterfeit regular and emergency messages accepted for analysis. The outcome of that analysis does not depend on the security of the shared secret, but the efficiency does. Our threat model assumes that sustained physical and non-physical attacks will trigger direction-finding and triangulation of the signal source, eventually eliminating the threat. The advantages of our proposed method are the following:

1. GF protocols are as convenient as public-key crypto, without having to manage keys, perform costly large-number computations on underpowered IoT devices, or be exposed to quantum attacks.

2. things can validate each other's transactions without trusting third parties.

3. even though the blocks are not mined, passive receivers of the authenticated block content (which we call witnesses) that monitor radio communications are able to detect wrongly accepted records and raise an alarm without the owner (Fog Server) being aware of the monitoring process.

4. validation proofs become objects in their own right, stored in the same CAS structure as all other blockchain records; they can be re-validated by any witness of the blockchain at low cost based solely on their content.

Future work will define mechanisms and protocols for managing trust whereby a thing may delegate verification of transactions to a blockchain witness.

\section{Acknowledgement}

Discussions with Bruce Christianson and his feedback are gratefully acknowledged.

\section{Authors' contributions}

The single author contributed $100 \%$. The author read and approved the final manuscript.

\section{Funding}

This work was supported in part by IMC corporation, Slovakia, under EU project BRAINE (Grant 876967)

\section{Availability of data and materials}

None produced, none available.

\section{Competing interests}

The author declares that he has no competing interests

Received: 20 August 2020 Accepted: 16 December 2020

Published online: 01 February 2021

\section{References}

Anderson R, Bergadano F, Crispo B, Lee J-H, Manifavas C, Needham R (1998) A new family of authentication protocols. SIGOPS Oper Syst Rev 32(4):9-20. https://doi.org/10.1145/302350.302353

Benet J (2014) IPFS - Content Addressed, Versioned, P2P File System. arXiv 1407.3561. http://arxiv.org/abs/1407.3561. Accessed 15 Aug 2020

Bernstein DJ, Hopwood D, Hülsing A, Lange T, Niederhagen R,

Papachristodoulou L, Schneider M, Schwabe P, Wilcox-O'Hearn Z (2015)

Sphincs: Practical stateless hash-based signatures. In: Oswald E, Fischlin M (eds). Advances in Cryptology - EUROCRYPT 2015. Springer, Berlin, Heidelberg. pp 368-397

Bonneau J, Miller A (2014) Fawkescoin. In: Christianson B, Malcolm J, Matyáš V, Švenda P, Stajano F, Anderson J (eds). Security Protocols XXII. Springer, Cham. pp 350-358

Crespo A, Garcia-Molina H (1998) Archival storage for digital libraries. In: Proceedings of the Third ACM Conference on Digital Libraries. Association for Computing Machinery, New York. pp 69-78. https://doi.org/10.1145/ 276675.276683

Danzi P, Kalør AE, Stefanovic C, Popovski P (2019) Delay and communication tradeoffs for blockchain systems with lightweight iot clients. IEEE Internet Things J 6(2):2354-2365

Dorri A, Kanhere SS, Jurdak R, Gauravaram P (2017) Blockchain for iot security and privacy: The case study of a smart home. In: 2017 IEEE International Conference on Pervasive Computing and Communications Workshops (PerCom Workshops). IEEE, Piscataway. pp 618-623

Espressif Systems (2020) ESP32 Technical Reference Manual. Available as https://www.espressif.com/sites/default/files/documentation/ esp32_technical_reference_manual_en.pdf. Accessed 15 Aug 2020

Haxhibeqiri J, Van den Abeele F, Moerman I, Hoebeke J (2017) Lora scalability: A simulation model based on interference measurements. Sensors 2017:1193. https://doi.org/10.3390/s17061193

Kouicem DE, Bouabdallah A, Lakhlef H (2018) Internet of things security: A top-down survey. Comput Netw 141:199-221

Lamport L (1979) Constructing digital signatures from a one-way function. Vol. 238. Technical Report CSL-98. Technical report

Leentvaar K, Flint J (1976) The capture effect in fm receivers. EEE Trans Commun 24(5):531-539

Liao C, Zhu G, Kuwabara D, Suzuki M, Morikawa H (2017) Multi-Hop LoRa Networks Enabled by Concurrent Transmission. IEEE Access 5:21430-21446 Merkle RC (1989) A certified digital signature. In: Proceedings on Advances in Cryptology (CRYPTO '89). Springer, Berlin, Heidelberg. pp 218-238

Quinlan S, Dorward S (2002) Venti: A new approach to archival storage. In: FAST Vol. 2. pp 89-101

Reyzin L, Reyzin N (2002) Better than biba: Short one-time signatures with fast signing and verifying. In: Batten L, Seberry J (eds). Information Security and Privacy. Springer, Berlin, Heidelberg. pp 144-153

Sentech Corporation (2019) LoRa and LoRaWAN: A Technical Overview. Technical report

Wang X, Zha X, Ni W, Liu RP, Guo YJ, Niu X, Zheng K (2019) Survey on blockchain for internet of things. Comput Commun 136:10-29

Zhu G, Liao C, Suzuki M, Narusue Y, Morikawa H (2018) Evaluation of LoRa receiver performance under co-technology interference. In: 2018 15th IEEE Annual Consumer Communications Networking Conference (CCNC). IEEE, Piscataway. pp 21430-21446

\section{Publisher's Note}

Springer Nature remains neutral with regard to jurisdictional claims in published maps and institutional affiliations.

\section{Submit your manuscript to a SpringerOpen ${ }^{\circ}$ journal and benefit from:}

- Convenient online submission

- Rigorous peer review

- Open access: articles freely available online

- High visibility within the field

- Retaining the copyright to your article

Submit your next manuscript at $\boldsymbol{\nabla}$ springeropen.com 